\title{
NONCOMMUTATIVE GEOMETRY AND APPLICATIONS TO ASTROPHYSICS
}

\author{
S.S. MOSKALIUK, ${ }^{1}$ M. WOHLGENANNT ${ }^{2}$ \\ ${ }^{1}$ Bogoliubov Institute for Theoretical Physics \\ (14-b, Metrolohichna Str., Kyiv 03680, Ukraine; e-mail: mss@bitp. kiev. ua) \\ ${ }^{2}$ Faculty of Physics, University of Vienna \\ (Boltzmanngasse 5, A-1090 Vienna, Austria; e-mail: michael.wohlgenannt@univie.ac.at)
}

PACS 95.30.Cq, 95.30.Sf (C) 2012 where $B_{i j}=-B_{j i}=$ const. In the limit of strong magnetic field, we project onto the lowest Landau level. As a result, we obtain a nonvanishing Dirac-bracket

$\left\{x^{i}, x^{j}\right\}_{\mathrm{DB}}=\frac{c\left(B^{-1}\right)^{i j}}{e}$. Some approaches to noncommutative gravity are reviewed. Furthermore, we discuss some applications to astrophysics.

\section{Noncommutative Geometry}

In noncommutative geometry, the coordinates are promoted to operators with nonvanishing commutation relations:

$\left[\hat{x}^{i}, \hat{x}^{j}\right]=\mathrm{i} \Theta^{i j}(\hat{x})$,

where $\Theta^{i j}=-\Theta^{j i}$ satisfies the Jacobi identity

$\left[\theta^{i j}(\hat{x}), \hat{x}^{k}\right]+\left[\theta^{k i}(\hat{x}), \hat{x}^{j}\right]+\left[\theta^{j k}(\hat{x}), \hat{x}^{i}\right]=0$.

The aim is to build models on such space-time structures and to study their properties. In this introductory section, we want to provide various motivations for studying noncommutative geometries and to introduce the basic mathematical notions. The most remarkable, in our opinion, is the intimate connection between noncommutative gauge theory and gravity. This connection is not fully understood at present and studied from different points of view (see, e.g., [1-6] and references therein for a merely exemplary list of quotations). In this talk, we want to review some approaches to noncommutative gravity and applications to astrophysics.

\subsection{Motivation}

Let us mention the Landau problem as the first example. Here, the noncommutative coordinates arise classically in a certain limit. Let us consider a particle with charge $e$ moving in a plane with a magnetic field normal to that plane. This situation is described by the action

$S=\int d t\left(\frac{1}{2} m \dot{x}_{i} \dot{x}^{i}-\frac{e}{c} B_{i j} x^{i} \dot{x}^{j}\right)$, a. Quantum gravity. Measuring a distance $l$ between two particles, we have to use photons of appropriate wavelength. An energy $E=\frac{h c}{\lambda} \sim \frac{h c}{l}$ has to be deposited in the measuring region. If $l$ is of the order of the Planck length $l_{\mathrm{Pl}}$,

$l \sim l_{P l}=\sqrt{\frac{\hbar G}{c^{3}}}$

the energy deposited is given by

$E=h c \sqrt{\frac{c^{3}}{\hbar G}}$

This corresponds to an inertia of the Planck mass:

$M=\frac{E}{c^{2}}=\frac{h}{c} \sqrt{\frac{c^{3}}{\hbar G}} \sim \sqrt{\frac{\hbar c}{G}}=M_{P l}$.

The associated Schwarzschild radius is given by $r_{S}=$ $\frac{2 G M}{c^{2}}=2 \sqrt{\frac{\hbar G}{c^{3}}}=2 l_{P l}$. This means that the measuring process creates a black hole. In order to avoid this scenario, the uncertainty relations for coordinates have to be assumed [7]. A natural way to achieve the uncertainty relations is via Eq. (1).

b. String theory. In the context of open strings with D-branes in a background magnetic field $B_{\mu \nu}$, the end points of the open strings are confined to the D-branes. The space-time dynamics is given by a noncommutative field theory in the low-energy limit [8-10]. 


\subsection{Coordinate algebra}

Let us discuss the commutation relations for the coordinates,

$\left[\hat{x}^{i}, \hat{x}^{j}\right]=\mathrm{i} \Theta^{i j}(\hat{x})$,

in more details. The structure constants $\Theta^{i j}(\hat{x})$ might be any functions of the generators with $\Theta^{i j}=-\Theta^{j i}$ and satisfy the Jacobi identity. Most commonly, the commutation relations are chosen to be constant, linear, or quadratic in the generators. In the canonical case, the relations are constant:

$\left[\hat{x}^{i}, \hat{x}^{j}\right]=\mathrm{i} \Theta^{\mathrm{ij}}=$ const.

The linear or Lie-algebra case

$\left[\hat{x}^{i}, \hat{x}^{j}\right]=\mathrm{i} \lambda_{\mathrm{k}}^{\mathrm{ij}} \hat{\mathrm{x}}^{\mathrm{k}}$

where $\lambda_{k}^{i j} \in \mathbb{C}$ are the structure constants, basically has been discussed in two different settings, namely fuzzy spaces and $\kappa$-deformation. The third commonly used choice is a quadratic commutation relation,

$\left[\hat{x}^{i}, \hat{x}^{j}\right]=\left(\frac{1}{q} \hat{R}_{k l}^{i j}-\delta_{l}^{i} \delta_{k}^{j}\right) \hat{x}^{k} \hat{x}^{l}$

where $\hat{R}_{k l}^{i j} \in \mathbb{C}$ is the so-called $\hat{R}$-matrix corresponding to quantum groups and is the solution of the quantum Yang-Baxter equation.

Independent of the explicit form of $\Theta^{i j}$, the commutative algebra of functions on space-time has to be replaced by the noncommutative algebra $\widehat{\mathcal{A}}$ generated by the coordinates $\hat{x}^{i}$, subject to the ideal $\mathcal{I}$ of relations generated by the commutation relations,

$\widehat{\mathcal{A}}=\frac{\mathbb{C}\left\langle\left\langle\hat{x}^{1}, \ldots \hat{x}^{n}\right\rangle\right\rangle}{\left[\hat{x}^{i}, \hat{x}^{j}\right]-\mathrm{i} \Theta^{i j}}$.

A noncommutative function $\hat{f}$ is given by a (formal) power series

$\widehat{\mathcal{A}} \ni \hat{f}(\hat{x})=\sum_{n=0}^{\infty} c_{i_{1} \ldots i_{n}}: \hat{x}^{i_{1}} \ldots \hat{x}^{i_{n}}:$,

where :: indicates an ordering for monomials in $\widehat{\mathcal{A}}$. In case of the normal ordering, i.e. coordinates with lower index to the left, this means, e.g.,

$: \hat{x}^{1} \hat{x}^{2} \hat{x}^{1}:=\left(\hat{x}^{1}\right)^{2} \hat{x}^{2}$

or, in case of the symmetric ordering,

$: \hat{x}^{1} \hat{x}^{2} \hat{x}^{1}:=\frac{1}{3}\left(\left(\hat{x}^{1}\right)^{2} \hat{x}^{2}+\hat{x}^{1} \hat{x}^{2} \hat{x}^{1}+\hat{x}^{2}\left(\hat{x}^{1}\right)^{2}\right)$.
In the latter situation, we can also use plane waves as the basis:

$\mathrm{e}^{\mathrm{i} k_{j} \hat{x}^{j}}$.

Let $\mathcal{A}$ be the commutative algebra of functions. Then there is an isomorphism mapping the commutative function algebra equipped with an additional noncommutative product $\star$ to the noncommutative one $\widehat{\mathcal{A}}$,

$W:(\mathcal{A}, \star) \longrightarrow(\widehat{\mathcal{A}}, \cdot)$,

This isomorphism exists, iff the noncommutative algebra together with the chosen basis (ordering) satisfies the so-called Poincaré-Birkhoff-Witt property, i.e. any monomial of order $n$ can be written as a sum of the basis monomials of order $n$ or smaller, by reordering and thereby using the algebra relations (1).

Let us stick to the most important example: the canonical deformation with $\Theta^{i j}(\hat{x})=$ const and the symmetric ordering: The Weyl-map is given by

$W: f(x) \mapsto \hat{f}(\hat{x}):=\int d^{4} k \tilde{f}(k) \mathrm{e}^{\mathrm{i} k_{j} \hat{x}^{j}}$

where

$f(k)=\frac{1}{(2 \pi)^{4}} \int d^{4} x f(x) \mathrm{e}^{-\mathrm{i} k_{j} x^{j}}$,

i.e., plane waves are mapped to plane waves,

$W: \mathrm{e}^{\mathrm{i} k_{j} x^{j}} \mapsto \mathrm{e}^{\mathrm{i} k_{j} \hat{x}^{j}}$.

The map $W$ establishes an isomorphism of vector spaces. In order to extend $W$ to an algebra isomorphism, we have to introduce a new noncommutative multiplication $\star$ in $\mathcal{A}$. This star product is defined by

$W(f \star g):=W(f) \cdot W(g)=\hat{f} \cdot \hat{g}$,

where $f, g \in \mathcal{A}, \hat{f}, \hat{g} \in \widehat{\mathcal{A}}$. We obtain

$W(f) W(g)=\int d^{4} k d^{4} p \tilde{f}(k) \tilde{g}(p) \mathrm{e}^{\mathrm{i} k_{j} \hat{x}^{j}} \mathrm{e}^{\mathrm{i} p_{j} \hat{x}^{j}}=$

$=\int d^{4} k d^{4} p \tilde{f}(k) \tilde{g}(p) \times \mathrm{e}^{-\frac{\mathrm{i}}{2} p_{m} \Theta_{m n} k_{n}} \mathrm{e}^{\mathrm{i}\left(k_{j}+p_{j}\right) \hat{x}^{j}}$

and applying $W^{-1}$ yields

$f \star g(x)=\int d^{4} k d^{4} p \tilde{f}(k) \tilde{g}(p) \mathrm{e}^{-\frac{i}{2} p_{m} \theta_{m n} k_{n}} \mathrm{e}^{\mathrm{i}\left(k_{j}+p_{j}\right) x^{j}}=$

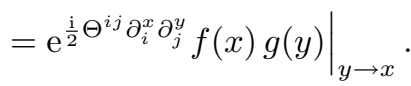


This is the so-called Moyal-Weyl star product, and $W$ is an algebra isomorphism, by construction,

$(\mathcal{A}, \star) \cong(\widehat{\mathcal{A}}, \cdot)$.

The information about the noncommutativity of $\widehat{\mathcal{A}}$ is encoded in the star product.

\subsection{Twists}

The Weyl-Moyal case is also the simplest example for a twist deformation:

$f \star g(x)=m \circ \mathcal{F}^{-1}(f \otimes g)$,

where $\mathcal{F}=e^{-\frac{i}{2} \theta^{i j} \partial_{i} \otimes \partial_{j}}$ denotes the so-called Drinfel'd twist. Let us introduce the useful Sweedler notation:

$\mathcal{F}=f^{\alpha} \otimes f_{\alpha}, \quad \mathcal{F}^{-1}=\bar{f}^{\alpha} \otimes \bar{f}_{\alpha}$,

where $f_{\alpha}, f^{\alpha}$ are some vector fields, and we imply a sum over $\alpha$. With this notation, we have

$f \star g(x)=\bar{f}^{\alpha}(f) \bar{f}_{\alpha}(g)$.

The so-called $\mathcal{R}$ matrix is defined by

$\mathcal{R}:=\mathcal{F}_{21} \mathcal{F}^{-1}=R^{\alpha} \otimes R_{\alpha}, \mathcal{R}^{-1}=\bar{R}^{\alpha} \otimes \bar{R}_{\alpha}$

with $\mathcal{F}_{21}=f_{\alpha} \otimes f^{\alpha}$. The $\mathcal{R}$ matrix "measures" the noncommutativity:

$f \star g=\bar{R}^{\alpha}(g) \star \bar{R}_{\alpha}(f)$.

Even more: The twist deforms the classical symmetry group $\mathcal{G}$ of the space - considered as the Hopf algebra $\mathcal{U} g=(h, \Delta, \epsilon, S)$, which is the universal enveloping algebra of its Lie algebra $g$. The Hopf algebra possesses dual structures. On the one hand, it is an algebra; on the other hand, it has a co-algebra structure consisting of a co-product $\Delta$ (dual to the normal multiplication), the so-called antipode, and a co-unit. These structure maps need to satisfy consistency conditions. For our purposes, the co-product $\Delta$ is most important. In case of the universal enveloping algebra of a Lie algebra $g$, the co-product is given by

$\Delta h=h \otimes \mathbf{1}+\mathbf{1} \otimes h, \quad h \in g$.

In the case of twisted symmetries, this co-product is modified in the following way:

$$
\begin{aligned}
\Delta: \mathcal{U} g & \rightarrow \mathcal{U} g \otimes \mathcal{U} g \\
\Delta & \rightarrow \Delta_{\mathcal{F}}=\mathcal{F} \Delta \mathcal{F}^{-1} \\
\mathcal{F} & \in \mathcal{U} g \otimes \mathcal{U} g
\end{aligned}
$$

using the twist defined in (20). It has to satisfy the cocycle condition, which guarantees the associativity of the $\star$-product.

Using the twist, all kinds of products can be deformed $[11,12]$. The star product between functions we had before is as follows:

$f \star g=\bar{f}^{\alpha}(f) \bar{f}_{\alpha}(g)$.

In the same way, we can consistently deform the product between a function $h$ and a vector field $v$

$h \star v \equiv \bar{f}^{\alpha}(h) \bar{f}_{\alpha}(v)$

or between tensor fields $\tau, \tau^{\prime}$ :

$\tau \otimes_{\star} \tau^{\prime}:=\bar{f}^{\alpha}(\tau) \otimes \bar{f}_{\alpha}\left(\tau^{\prime}\right)$,

where $\bar{f}^{\alpha}(\cdot)$ is understood as the classical Lie derivative with respect to the vector field $\bar{f}^{\alpha}$.

\section{Twisted Gravity}

Using the technique of twist deformation introduced before, let us define the $\star$-Lie derivative, i.e., infinitesimal diffeomorphisms:

$\mathcal{L}_{u}^{\star}(h):=\bar{f}^{\alpha}(u)\left(\bar{f}_{\alpha}(h)\right)$.

The space of vector fields equipped with the $\star$-Lie bracket

$\mathcal{L}_{u}^{\star} \mathcal{L}_{v}^{\star}-\mathcal{L}_{\bar{R}^{\alpha}(v)}^{\star} \mathcal{L}_{\bar{R}_{\alpha}(u)}^{\star}=: \mathcal{L}_{[u, v]_{\star}}^{\star}$,

where $[u, v]_{\star}=u \star v-\hat{R}^{\alpha}(v) \star \hat{R}_{\alpha}(u)$, defines the $\star$-Lie algebra of vector fields, denoted by $\Xi_{\star}$. Following [11, 12], we can introduce the notions of twisted Riemannian geometry. The star-connection $\nabla^{\star}$

$\nabla_{u}^{\star}: \Xi_{\star} \rightarrow \Xi_{\star}$

is defined to satisfy the axioms

$\nabla_{u+v}^{\star} z=\nabla_{u}^{\star} z+\nabla_{v}^{\star} z$

$\nabla_{h \star u}^{\star} v=h \star \nabla_{u}^{\star} v$

$\nabla_{u}^{\star}(h \star v)=\mathcal{L}_{u}^{\star}(h) \star v+\bar{R}^{\alpha}(h) \star \nabla_{\bar{R}_{\alpha}(u)}^{\star} v$,

where $u, v, z \in \Xi_{\star}, h$ is a function. Next, we define connection coefficients by

$\nabla_{\mu}^{\star} \hat{\partial}_{\nu}:=\Gamma_{\mu \nu}^{\sigma} \star \hat{\partial}_{\sigma}$ 
using the basis $\left\{\hat{\partial}_{\mu}\right\}$ of $\Xi_{\star}$. The torsion and curvature tensors are given by

$$
\begin{aligned}
& T^{\star}(u, v)=\nabla_{u}^{\star} v-\nabla_{\bar{R}^{\alpha}(v)}^{\star} \bar{R}_{\alpha}(u)-[u, v]_{\star}, \\
& R^{\star}(u, v, z) \equiv R^{\star}(u, v) z= \\
& =\nabla_{u}^{\star} \nabla_{v}^{\star} z-\nabla_{\bar{R}^{\alpha}(v)}^{\star} \nabla_{\bar{R}_{\alpha}(u)}^{\star} z-\nabla_{[u, v]_{\star}}^{\star} z,
\end{aligned}
$$

or, in components,

$$
T\left(\hat{\partial}_{\mu}, \hat{\partial}_{\nu}\right)=T_{\mu \nu}^{\rho} \hat{\partial}_{\rho}
$$

$$
R\left(\hat{\partial}_{\mu}, \hat{\partial}_{\nu}, \hat{\partial}_{\rho}\right)=R_{\mu \nu \rho}^{\sigma} \hat{\partial}_{\sigma}
$$

As in the commutative case, the Ricci tensor is defined by the following contraction of indices:

$R i c_{\mu \nu}=R_{\rho \mu \nu}^{\rho}$

The metric is a $\star$-symmetric tensor

$g^{\star}=g_{\mu \nu}^{\star} \star d \hat{x}^{\mu} \otimes_{\star} d \hat{x}^{\nu}$

The components of the inverse metric are determined with respect to the star product,

$g^{\star \mu \nu} \star g_{\nu \rho}^{\star}=g_{\rho \nu}^{\star} \star g^{\star \nu \mu}=\delta_{\nu}^{\mu}$.

A noncommutative metric is said to be compatible with the star-connection, if

$\nabla_{u}^{\star} g^{\star}=0, \forall u \in \Xi_{\star}$

Therefore, given a metric $g^{\star}$, one can consistently define Christoffel symbols $\Gamma_{j k}^{\star i}$, a compatible connection $\nabla^{\star}$, and the Riemann $R_{i j k}^{\star}{ }^{l}$ and Ricci tensors $R i c_{a b}^{\star}$.

Then the deformed Einstein equation has the familiar form

$R_{a b}^{\star}-\frac{1}{2} g_{a b}^{\star} \star R^{\star}=8 \pi G_{N} T_{a b}^{\star}$

Of special interest are Einstein spaces, where the deformed Ricci tensor is proportional to the noncommutative metric,

$R_{\mu \nu}^{\star}=c g_{\mu \nu}^{\star}$

\section{Noncommutative Astrophysics}

In this section, we want to present a brief review and to discuss the noncommutative Einstein equations (35). The equations are fairly complicated, since they involve the star product. Therefore, only very few explicit solutions are known.

In the simplest case, the twist consists of Killing vectors, i.e., of vector fields $K_{\alpha}$ with $\mathcal{L}_{K_{\alpha}} g^{\star}=0$. Let us denote the Lie algebra of (affine) Killing vector fields by $\mathbf{g}_{K}$. Then the twist is an element of the tensor product of the respective universal enveloping algebras,

$\mathcal{F} \in \mathcal{U} \mathrm{g}_{K} \otimes \mathcal{U} \mathrm{g}_{K}$

and the deformed Einstein equations are reduced to the undeformed ones [13].

Some explicit examples are studied in $[14,15]$, where we have showed that the deformed Einstein spaces (the Weyl-Moyal plane and the twisted sphere) are noncommutative Einstein spaces.

Schupp and Solodukhin [16] constructed a spherical symmetric vacuum solution (black hole) in $(3+1) \mathrm{D}$, by using the fuzzy sphere as the underlying noncommutative space:

$\left[\hat{x}^{a}, \hat{x}^{b}\right]=\mathrm{i} \lambda(\hat{\rho}) \epsilon_{a b c} \hat{x}^{c}$,

where $a, b, c=1,2,3$, and $\lambda$ denotes a function of the Casimir operator $\hat{\rho}=\hat{x}^{2}+\hat{y}^{2}+\hat{z}^{2}$ in the coordinate algebra. The corresponding twist is given by

$\mathcal{F}=\sum_{n} C_{n}\left(\frac{\lambda}{\rho}\right) \mathcal{L}_{\xi_{+}}^{n} \otimes \mathcal{L}_{\xi_{-}}^{n}$,

where $\xi_{ \pm}$are rotation generators. Since the rotation generators are Killing vectors, the solution is the classical one except for the parameters (eigenvalues of $\hat{\rho}$ ) $\rho=n \lambda$, $n=2 j=0,1,2, \ldots$ :

$g_{00}=\frac{a}{r}-1, \quad g_{0, i}=0, \quad g_{i j}=\frac{r^{2}}{\rho^{2}} \delta_{i j}$,

where $r=\frac{1}{\rho}\left(\rho+\frac{a}{4}\right)$. Some remarks are in order:

- The above solution is valued outside the horizon $r=a$ or $\rho=a / 4$. Therefore, $n>a /(4 \lambda)$.

- The noncommutative space-time is sliced by fuzzy spheres with radius $\rho$.

- Inside the horizon, they have found a different solution:

$g_{00}=1-\frac{a}{r}, \quad g_{0, i}=0, \quad g_{i j}=\frac{r^{2}}{\rho^{2}}\left(\delta_{i j}-\frac{x_{i} x_{j}}{\rho^{2}}\right)$, 
where $\rho^{2}=\frac{a^{2}}{2 r} \frac{1}{a-r}$.

The classical singularity, $r=0$, corresponds to $\rho=\infty$. Therefore, the singularity does not disappear, but it is replaced by a sequence of fuzzy spheres. One hope of noncommutative geometry is that singularities are dissolved. However, in this case, this is not true.

Ohl and Schenkel [17] discuss more general twists of the form

$\mathcal{F}=\exp \left(-\frac{\mathrm{i} h}{2} \theta^{a b} X_{a} \otimes X_{b}\right)$,

$\theta^{a b}=$ const and $X_{a}, X_{b}$ any vector fields satisfying

$\left[X_{a}, X_{b}\right]=0$

Starting from symmetry arguments, they classified the possible twists leading to deformed black hole models (spherical symmetric) and to deformed cosmological (Friedman-Robertson-Walker) models.

Let us turn to applications of noncommutative ideas to astrophysics. If the noncommutativity exists in the Nature, it seems that its effects can be observed more easily in astrophysical scenarios, than in collider experiments. The former always include huge distances, and small effects can add up. The noncommutative theories lead, in general, to modified dispersion relations of the kind

$E^{2}=p^{2} c^{2}(1+\lambda E)^{2}+m^{2} c^{4}$

where $\lambda$ is the noncommutative deformation parameter. Bertolami and Zarro [18] used the modified dispersion relation (43) in order to calculate corrections to the energy and the particle density, as well as to the pressure of a photon gas, a nonrelativistic ideal gas, and a degenerate fermion gas. These results are applied to study the stability of stars. There, the gas pressure and the radiation one

$P=P_{\text {rad }}+P_{\text {gas }}$

are in equilibrium with the gravitational force. Let us choose $\lambda=2.5 \times 10^{-19} \mathrm{GeV}^{-1}$. Then the following results for the ratio $P_{\mathrm{NC}} / P_{\mathrm{C}}$ hold (the index distinguishes between the noncommutative and the commutative case):

- Main sequence stars: $\frac{P_{\mathrm{NC}}}{P_{\mathrm{C}}}=10^{-25}$.

- White dwarfs: $\frac{P_{\mathrm{NC}}}{P_{\mathrm{C}}}=10^{-22}$.

- Neutron stars: $\frac{P_{\mathrm{NC}}}{P_{\mathrm{C}}}=10^{-19}$.
This means that the stability regions of stars are enlarged. The effect increases with the mass of stars. This calculation may not be very realistic, since it relies on many approximations and assumptions. But it points into the right direction and gives some hints what the effects of noncommutativity could be.

Another example is provided by $\gamma$-ray bursts in active galaxies (see, e.g., [19]). Due to the modified dispersion relations (43), there is a difference in the arrival time of photons with different energies:

$|\delta t| \approx \lambda \frac{L}{c} \delta \omega$

where $L$ denotes the distance of the galaxy, and $\delta \omega$ is the energy range of the burst. So far, an effect like this has not been detected. This leads to bounds for the noncommutativity parameter $\lambda$.

\section{Noncommutative Topological Quantum Field Theory}

Atiyah's axioms for an $n$-dimensional topological quantum field theory (TQFT) describe it as a symmetric monoidal functor

$Z: \mathbf{n C o b} \rightarrow$ Hilb.

One value of TQFTs is that they provide invariants of manifolds and, in particular, of 3-manifolds (potentially with boundary). This is closely related to the subject of knot theory, since knots are studied by their complement in some 3-manifold. In particular, in the codimension-2 case, we replace $\mathbf{n C o b i}$ by $\mathbf{n C o b}$ [25], a double bicategory, whose 2-morphisms are cobordisms between cobordisms. We have also passed from Vect, the category of vector spaces and linear maps, to 2Vect, a double bicategory, whose objects are 2 -vector spaces, whose morphisms are 2-linear maps (linear functors) and whose 2-morphisms are natural transformations.

In general, a " $k$-tuply extended TQFT" assigns highercategorical structures called $k$-vector spaces to manifolds of codimension $k$. In particular, it is a (weak, monoidal) $k$-functor [25]:

$Z: \mathbf{n C o b}_{\mathbf{k}} \rightarrow \mathbf{k}-$ Vect.

Here, $\mathbf{n C o b}_{\mathbf{k}}$ is a $k$-category (that is, a higher category having $j$-morphisms for $j=1 \ldots k$ ), whose objects are $(n-k)$-dimensional manifolds, and whose $j$-morphisms are $(n-k+j)$-dimensional cobordisms between $(j-1)$ morphisms. That is, they are manifolds with corners, 
which have the source and target cobordisms as boundary components. The $k$-category $\mathbf{k}-$ Vect is a highercategorical analog of Vect in an appropriate sense. In our case, the extended topological quantum field theory is a double functor $Z$ from the double bicategory $C M(d)$ of $d$-dimensional manifolds to the double bicategory of $H$ of (usually Hermitian) finite dimensional (noncommutative) vector spaces, and some axioms are satisfied [25].

Thus, the extended topological quantum field theory in dimension $d$ is a functor

\section{$Z: \mathbf{C}(\mathbf{d}) \rightarrow \operatorname{Morph}(\mathbf{H})$}

between double bicategories such that:

(1) the disjoint union in $\mathbf{C}(\mathbf{d})$ goes to the tensor product

$\cup \mapsto \otimes$,

where $\left({ }_{-}\right)^{*}: H \rightarrow H^{\circ}$ is a dualization of vector spaces.

(2) changing the orientation in $\mathbf{C}(\mathbf{d})_{\mathbf{0}}$ goes to the dualization

$(-) \mapsto(.)^{*}$

Thus, as a consequence of double bicategorical functorial properties, we get

(1) for each compact closed oriented smooth ddimensional manifold $X \in \operatorname{Obj}\left(\mathbf{C}(\mathbf{d})_{\mathbf{0}}\right)$, the value of the functor $Z(X)$ is a finite dimensional (noncommutative) vector space over the field $\mathbf{C}$ of complex numbers (usually with the Hermitian metric),

(2) for each $(Y, f): X \Rightarrow X^{\prime}$ from $\operatorname{Obj}\left(\mathbf{C}(\mathbf{d})_{1}\right)$, the value of the functor $Z(Y, f)$ is a homomorphism $Z(X) \rightarrow Z\left(X^{\prime}\right)$ of (Hermitian) (noncommutative) vector spaces,

and the following well-known axioms of the extended topological quantum field theory are satisfied:

A(1) (involutivity) $Z(-X)=Z(X)^{*}$, where $-X$ denotes the manifold with opposite orientation, and * denotes the dual (noncommutative) vector space.

$\mathrm{A}(2)$ (multiplicativity) $Z\left(X \cup X^{\prime}\right)=Z(X) \otimes Z\left(X^{\prime}\right)$, where $\cup$ denotes a disconnected union of manifolds.

$\mathrm{A}(3)$ (associativity) For the composition $\left(Y^{\prime \prime}, f^{\prime \prime}\right)=$ $(Y, f) *\left(Y^{\prime}, f^{\prime}\right)$ of cobordisms,

$Z\left(Y^{\prime \prime}, f^{\prime \prime}\right)=Z\left(Y^{\prime}, f^{\prime}\right) \circ Z(Y, f) \in \operatorname{Hom}_{\mathbf{C}}\left(Z(X), Z\left(X^{\prime \prime}\right)\right)$.
(Usually the identifications

$Z\left(X^{\prime}-X\right) \cong Z(X)^{*} \otimes Z\left(X^{\prime}\right) \cong \operatorname{Hom}_{\mathbf{C}}\left(Z(X), Z\left(X^{\prime}\right)\right)$

allow us to identify $Z(Y, f)$ with the element $Z(Y, f) \in Z(\partial Y)$.

$\mathrm{A}(4)$ For the initial object, $\emptyset \in \operatorname{Obj}\left(\mathbf{C}(\mathbf{d})_{\mathbf{0}}\right) \quad \mathbf{Z}(\emptyset)=\mathbf{C}$.

$\mathrm{A}(5)$ (trivial homotopy condition) $Z(X \times[0,1])=$ $\operatorname{id}_{Z(X)}$.

\section{Summary}

In our opinion, noncommutative ideas can best be tested in astrophysical scenarios. Up to now, there are some predictions, but a contact to "real" physics and experiments is not yet established. The presented model of twisted gravity is an interesting candidate implementing the noncommutativity into gravitational physics. However, matter has not been included so far and resembles a further step in the development. This might allow the study of effects like the cosmic microwave background or Hawking radiation within this framework.

The authors are especially grateful to the Austrian Academy of Sciences and the Russian Foundation for Fundamental Research which co-financed this research in the framework of the collaboration with the National Academy of Sciences of Ukraine.

The authors also could have not succeeded in pursuing this program without the collaborations for many years with A.Z. Petrov, W. Kummer, and J. Wess.

1. R.J. Szabo, Class. Quant. Grav. 23, R199 (2006); http://arxiv.org/abs/hep-th/0606233.

2. H.S. Yang, Int. J. Mod. Phys. A 24, 4473 (2009); http://arxiv.org/abs/hep-th/0611174.

3. X. Calmet and A. Kobakhidze, Phys. Rev. D. 74, 047702 (2006); http://arxiv.org/abs/hep-th/0605275.

4. H. Steinacker, JHEP 12, 049 (2007), http://arxiv.org/abs/arXiv:0708.2426[hep-th].

5. M. Chaichian, A. Tureanu, and G. Zet, Corrections to Schwarzschild Solution in Noncommutative Gauge Theory of Gravity, http://dx.doi.org/10.1016/ j.physletb.2008.01.029; Phys. Lett. B 660, 573 (2008); http://arxiv.org/abs/0710.2075[hep-th].

6. A.P. Balachandran, A. Pinzul, B.A. Qureshi, and S. Vaidya, Phys. Rev. D 76105025 (2007); http://arxiv.org/abs/arXiv:0708.0069[hep-th].

7. S. Doplicher, K. Fredenhagen, and J.E. Roberts, Commun. Math. Phys. 172, 187 (1995); http://arxiv.org/abs/hep-th/0303037. 
8. A. Connes, M.R. Douglas, and A.S. Schwarz, JHEP 02, 003 (1998); http://arxiv.org/abs/hep-th/9711162.

9. N. Seiberg and E. Witten, JHEP 09, 032 (1999); http://arXiv.org/abs/hep-th/9908142.

10. V. Schomerus, JHEP 06, 030 (1999); http://arxiv.org/abs/hep-th/9903205.

11. P. Aschieri, C. Blohmann, M. Dimitrijevic, F. Meyer, P. Schupp, and J. Wess, Class. Quant. Grav. 22, 3511 (2005); http://arxiv.org/abs/hep-th/0504183.

12. P. Aschieri, M. Dimitrijevic, F. Meyer, and J. Wess, Class. Quant. Grav. 23, 1883 (2006); http://arxiv.org/abs/hep-th/0510059.

13. P. Aschieri and L. Castellani, Noncommutative Gravity Solutions, http://arxiv.org/abs/0906.2774.

14. P. Aschieri and M. Wohlgenannt, Noncommutative Einstein Spaces, work in preparation.

15. S.S. Moskaliuk and M. Wohlgenannt, Ukr. J. Phys. 55, 505 (2010).

16. P. Schupp and S. Solodukhin, Exact Black Hole Solutions in Noncommutative Gravity, http://arxiv.org/abs/ 0906.2724 .

17. T. Ohl and A. Schenkel, Cosmological and Black Hole Spacetimes in Twisted Noncommutative Gravity, http://dx.doi.org/10.1088/1126-6708/2009/10/052; JHEP 10, 052 (2009); http://arxiv.org/abs/0906.2730.

18. O. Bertolami and C.A.D. Zarro, Towards a Noncommutative Astrophysics, http://dx.doi.org/ 10.1103/PhysRevD.81.025005; Phys. Rev. D 81, 025005 (2010); http://arxiv.org/abs/0908.4196.
19. G. Amelino-Camelia and S. Majid, Int. J. Mod. Phys. A 15, 4301 (2000); http://arxiv.org/abs/hep-th/9907110.

20. J.C. Morton, Extended TQFT, Gauge Theory, and 2Linearization, Preprint, math.QA/1003.5603 (2010).

21. M.F. Atiyah, Publ. Math. Inst. Hautes Etudes Sci. Paris 68, 175 (1989).

22. S.S. Moskaliuk and T.A. Vlassov, Ukr. J. Phys. 43, 836 (1998).

23. S.S. Moskaliuk and A.T. Vlassov, in Proceedings of the 5th Wigner Symposium (World Sci., Singapore, 1998), p. 162.

24. S.S. Moskaliuk, From Cayley-Klein Groups to Categories (TIMPANI, Kyiv, 2006).

25. S.S. Moskaliuk and N.M. Moskaliuk, J. of Phys.: Conf. Ser. 343, 012080 (2012).

Received 22.01.09

\section{НЕКОМУТАТИВНА ГЕОМЕТРІЯ ТА ЗАСТОСУВАННЯ} В АСТРОФІЗИЦІ

М. Волгенант, С.С. Москалюк

$\mathrm{P}$ е $з$ ю м е

Наведено основні концепції некомутативної геометрії та некомутативної топологічної квантової теорії поля. Дано огляд деяких підходів до некомутативної гравітації. Також обговорено застосування некомутативної геометрії в астрофізиці. 